Supplemental information

for

\title{
DNA Strands Trigger the Intracellular Release of Drugs from Mucin-Based Nanocarriers
}

Ceren Kimna', Theresa Monika Lutz', Hongji Yan², Jian Song1, Thomas Crouzier', and Oliver Lieleg ${ }^{\#, 1}$

${ }^{1}$ Department of Mechanical Engineering and Munich School of Bioengineering, Technical University of Munich, Boltzmannstraße 11, 85748 Garching, Germany

${ }^{2}$ Division of Glycoscience, Department of Chemistry, School of Engineering Sciences in Chemistry, Biotechnology and Health, KTH Royal Institute of Technology, AlbaNova University Center, 10691 Stockholm, Sweden

"Corresponding author

Prof. Dr. Oliver Lieleg

Department of Mechanical Engineering and Munich School of Bioengineering, Technical University of Munich,

Boltzmannstraße 11, 85748 Garching, Germany

e-mail: oliver.lieleg@tum.de,

phone: +49 89289 10952, fax: + 498928910801 


\section{Mucin purification and modification with DNA}

\subsection{Mucin purification}

Porcine gastric mucin MUC5AC was purified manually as described previously. ${ }^{1}$ In brief, gastric mucus was obtained from gently rinsed pig stomachs by manual scraping the surface of the gastric tissue. The collected mucus was diluted 5-fold in a sodium phosphate buffer $(10 \mathrm{mM}, \mathrm{pH}=7.0)$ containing $170 \mathrm{mM} \mathrm{NaCl}$ and $0.04 \%(\mathrm{w} / \mathrm{v})$ sodium azide (Carl Roth, Karlsruhe, Germany) and stirred at $4{ }^{\circ} \mathrm{C}$ overnight. Cellular debris was removed via two centrifugation steps (first run: $8300 \mathrm{~g}$ at $4{ }^{\circ} \mathrm{C}$ for $30 \mathrm{~min}$; second run: $15000 \mathrm{~g}$ at $4{ }^{\circ} \mathrm{C}$ for $45 \mathrm{~min})$, and a final ultracentrifugation step (150000 $\mathrm{g}$ at $4{ }^{\circ} \mathrm{C}$ for $\left.1 \mathrm{~h}\right)$. Subsequently, the mucins were purified by size exclusion chromatography using an ÄKTA purifier system (GE Healthcare, Munich, Germany) and an XK50/100 column packed with Sepharose 6FF. The obtained mucin fractions were pooled, dialyzed against ultrapure water, and concentrated by cross-flow filtration. The concentrates were then lyophilized and stored at $-80^{\circ} \mathrm{C}$ until further use. With this purification procedure, highly functional mucin is obtained; however, our mucin samples also contain small amounts of protein impurities as well as mucinassociated DNA.

\subsection{Mucin structure}

The mucin is a large glycoprotein. Its terminal ends are partially folded, whereas the central region is extended due to their highly glycosylation. These glycans constitute about twothirds of the molar mass of the molecule. Some of these carbohydrates contain negatively charged sulfate groups and sialic acids, which render the mucin molecule overall polyanionic (Fig. S1).

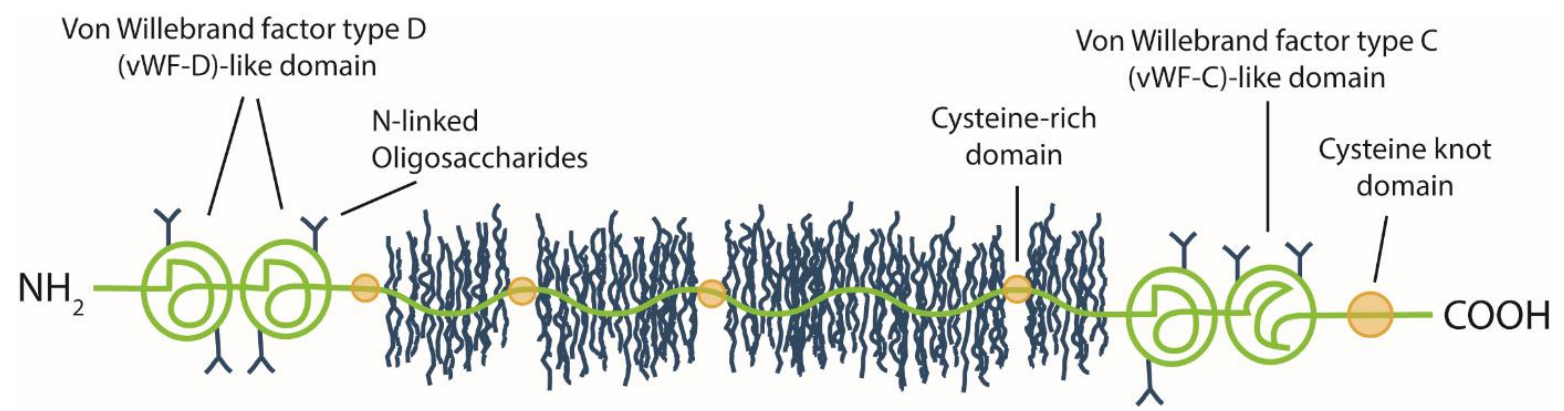

Figure S1: Schematic representation of the mucin structure. 
In the main paper, to investigate the reversibility of the condensation process, which creates nanoparticles from mucin macromolecules, the size of condensed mucins is investigated in different states (i.e., condensed and subjected to different $\mathrm{pH}$ levels, enzymatic environments, or triggered) by dynamic light scattering (DLS). For comparison, we here show similar size DLS measurements (Table S1) conducted with mucins from a freshly prepared solution (without any condensation agent). Therefore, freeze-dried mucin was solubilized in $\mathrm{ddH}_{2} \mathrm{O}(0.1 \mathrm{mg} / \mathrm{mL})$ and then analyzed as in the main paper.

Table S1:DLS results obtained for a freshly prepared mucin solution $(0.1 \mathrm{mg} / \mathrm{mL})$

\begin{tabular}{|c|c|c|c|}
\hline & $\begin{array}{c}\text { Intensity-average } \\
\text { hydrodynamic size [nm] }\end{array}$ & Area [\%] & $\begin{array}{c}\text { Polydispersity Index } \\
\text { (PDI) }\end{array}$ \\
\hline Peak 1 & 1523 & 87.2 & 0.74 \\
\hline Peak 2 & 25.22 & 12.8 & \\
\hline
\end{tabular}

\subsection{Fluorescence labeling of mucin}

A carboxy modified ATTO dye (Atto488, ATTO-TEC GmbH, Siegen, Germany) was conjugated to mucin via carbodiimide chemistry. In brief, $5 \mathrm{mM}$ EDC and $5 \mathrm{mM}$ sulfo-NHS were added to the dye solution ( $\mathrm{C}_{\mathrm{ATTO}}=1.0 \mathrm{mg} / \mathrm{mL}$ in MES buffer $(10 \mathrm{mM}, \mathrm{pH}=5)$ ) and incubated at room temperature in a light excluded environment for $3 \mathrm{~h}$. This extended incubation time ensured that the remaining free EDC was hydrolyzed before mucin was added to avoid crosslinking of the mucin molecules. In parallel, $40 \mathrm{mg}$ of purified MUC5AC was dissolved in $19 \mathrm{~mL}$ PBS $(10 \mathrm{mM}, \mathrm{pH}=7)$. Then, the solutions were thoroughly mixed and again allowed to react at $\mathrm{RT}$ for $3 \mathrm{~h}$. To remove unbound dye molecules, the mixture was dialyzed against ultrapure water for 2 days (molecular weight cut-off $=300 \mathrm{kDa}$ ). The labeled mucins were then lyophilized and stored at $-80^{\circ} \mathrm{C}$ until further use.

\subsection{Verifying the specificity of DNA/mucin interactions}

For the detection of mucin glycoproteins, SDS gels were stained with a PAS (periodic acid/Schiff solution) set. In brief, after DNA and Coomassie staining, the gel was placed into an $0.5 \%$ periodic acid solution (Carl Roth, Germany) and incubated for 7 min while shaking at RT. Afterwards, the gel was rinsed with distilled water and carefully transferred into 100 $\mathrm{mL}$ of Schiff's reagent (Carl Roth, Germany). After incubation for $15 \mathrm{~min}$, the gel was rinsed 
with tap water and then imaged. Here, mucin was stained in a light pink color as shown in the bands of columns 3, 4 and 5 (Fig. S2).

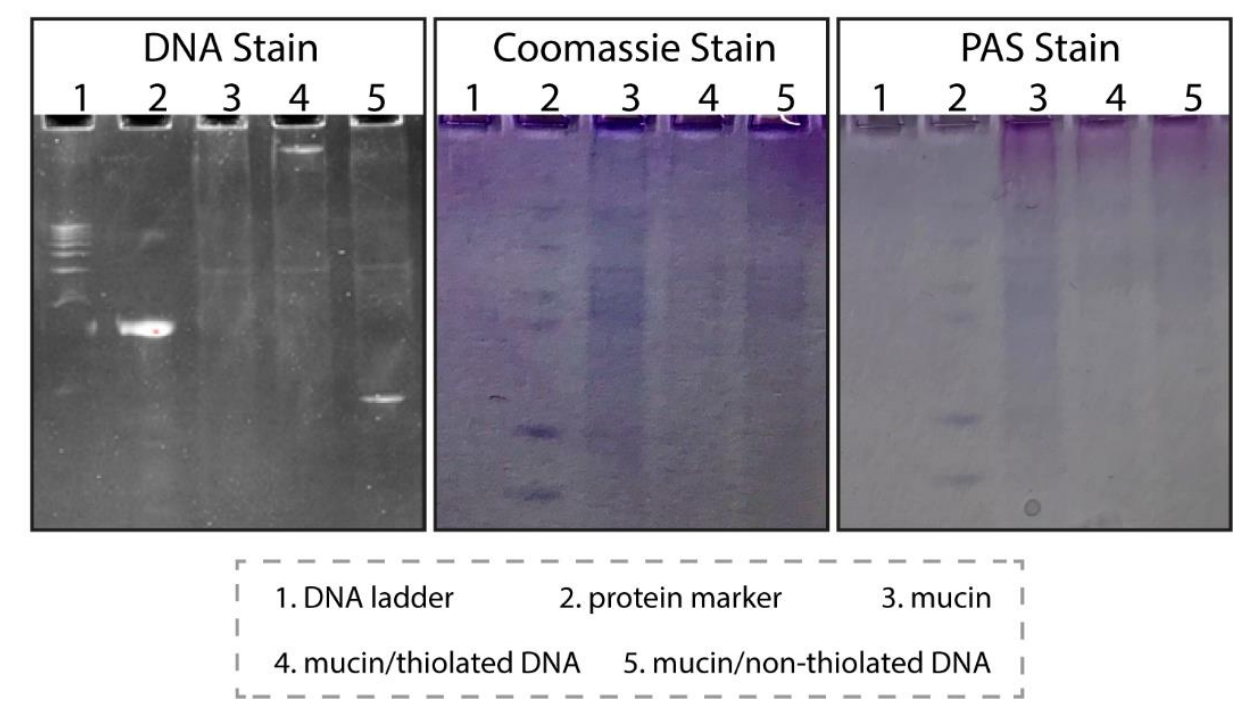

Figure S2: Electrophoretic separation of triple-stained mucin and DNA/mucin mixtures. The same gel is imaged after DNA, Coomassie and PAS staining to show DNA, protein and glycoprotein (= mucin) components, respectively.

\section{Characterization of mucin-based NPs}

\subsection{Determining the amount of crDNA conjugated to mucin and the amount of dDNA needed for triggering NP dispersal}

First, mucin was solubilized at a concentration of $1 \mathrm{mg} / \mathrm{mL}$ and incubated with the crDNA strands. Then, unmodified and PLL-coated mucin NPs were prepared as described in the main text. Unbound crDNA molecules were removed by dialyzing the samples against $\mathrm{dd}_{2} \mathrm{O}$ for 2 days. Afterwards, the DNA content of the mucin-NP samples was measured spectrophotometrically (Nanodrop-1000, Thermo Fischer Scientific, Ulm, Germany).

Since the manually purified is associated with DNA (content $\sim 5 \mathrm{ng} / \mu \mathrm{L}$ ), this value was subtracted from those obtained from crDNA conjugated mucin samples ( $c_{\mathrm{crDNA}}=\sim 40 \mathrm{ng} / \mu \mathrm{L}$, Fig. S3). With this approach, we determined that $~ 13$ crDNA molecules can bind to a single mucin molecule. With the same strategy, we found that the PLL coating did not affect the crDNA content; this was expected since the crDNA conjugation was performed prior to PLL coating. 


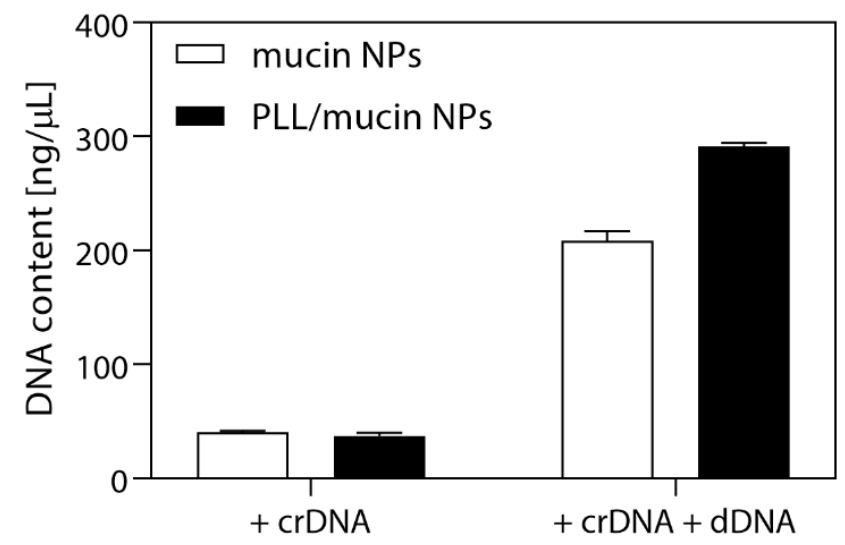

Figure S3: single-stranded DNA content of mucin and PLL/mucin NPs after crosslinking and dDNA incorporation. Data shown represent mean values, error bars denote the standard deviation as obtained from $n=3$ independent samples.

The protein sequence of MUC5AC (the mucin molecule used in this study) was retrieved from the database UniProtKB (https://www.uniprot.org, 25th September 2018, Accession number: A0A287ANG4). From this sequence, we can estimate that a single mucin molecule comprises 270 cysteine; however, 109 of them are located in the glycosylated region of the glycoprotein, where they are unlikely to interact with the crDNA strands due to a combination of electrostatic repulsion and steric effects. The remaining cysteines are located in the terminal regions $(91+70=161$ in total) of the mucin macromolecule; however, also there, not all of them are accessible for disulfide bond formation (due to the presence of folded polypeptide strands in the vWF-C-like and vWF-D-like domains). By excluding these domains, we conclude that $\sim 98$ cysteines from the mucin polypeptide backbone could be accessible in total. With this value, we can calculate the minimal conjugation efficiency of mucin/crDNA to be 13 DNA/98 cysteines (which corresponds to $\sim 13 \%)$.

Next, the trigger DNA (dDNA) was added to the crDNA-crosslinked mucin NPs, and excessive dDNA (that did not interact with the mucin NPs) was removed by dialysis. After 2 days of dialysis, the DNA concentration of the samples was determined as described above. With this approach, we measure $\sim 75 \pm 4$ dDNA molecules bound per mucin molecule, which corresponds to $12 \mathrm{dDNA}$ strands per crDNA/crDNA complex. For the PLL-coated mucin NPs, the corresponding value was found to be higher, i.e., $\sim 21 \pm 1$ dDNA strands per crDNA/crDNA cross-link. We interpret this finding such that electrostatic attraction forces 
between the cationic coating of the NPs and the negatively charged DNA trap dDNA strands on the NP surface.

\subsection{Stability of mucin-based NPs}

We analyzed the stability of DNA-cross-linked mucin NPs when subjected to different buffer conditions that mimic distinct, physiologically relevant challenges. In this particular set of experiments, we tracked two parameters describing the mucin NP population: the average NP size and the derived count rate, the latter of which is a function of the number of intact NPs. First, we incubated the DNA-stabilized mucin-based NPs at two different pH levels, i.e., at 7.4 and 5.2. Those values were chosen as they represent cytoplasmic and endosomal pH levels, respectively. Importantly, we found that even after extended incubation for 14 days at $37^{\circ} \mathrm{C}$, the mucin-based NPs maintained their condensed configuration and the derived count rate remained virtually constant as well (Fig. S4a,b). At the same time, the polydispersity index (PDI) of the NPs showed only little variation (Table S2), which indicates moderately homogeneous size distributions and the presence of only a few aggregates. ${ }^{2}$

Of course, in an intracellular environment, the mucin-based NPs will also be exposed to enzymatic attack. Since the NPs are formed from a proteinous component (mucin), we challenged the DNA-stabilized mucin-based NPs with trypsin. After NP exposure to this enzyme, we observed a slow decrease in the derived count rate over time (Fig. S4c). At the same time, the size of the mucin-based NPs remained relatively constant during the first 5 days and then started to decrease as well. This suggests that the mucin-based NPs are somewhat vulnerable towards enzymatic digestion with trypsin. Previously, we demonstrated that a trypsin treatment of mucins mainly removes the terminal domains of the glycoprotein but leaves the glycosylated central region of mucins largely intact. ${ }^{3}$ With this information in mind, both the decrease in the count rate and average size of mucinbased NPs can be interpreted as gradual enzymatic digestion of the NPs. This slow degradation is probably initiated at the termini of the mucin glycoproteins. However, the protease seems not to be able to easily attack mucin/mucin cross-links established via crDNA linked to cysteine residues of the mucin backbone.

Those DNA cross-links should, however, be degradable by DNasel, since this enzyme can attack the DNA backbone by catalyzing the hydrolytic cleavage of phosphodiester linkages (Fig. S4d). Indeed, when the mucin-based NPs were exposed to a high concentration of this 
enzyme ( $50 \mu \mathrm{g} / \mathrm{mL}$, grey shaded area), we found a clear response of the NPs: after only $4 \mathrm{~h}$ of incubation, we observe a rapid size increase. However, whereas this proves once more that it is indeed the DNA-based cross-links that stabilize the mucin-based NPs, this DNasel concentration is much higher than the average levels present in human serum (which are in the range of $3-24 \mathrm{~g} / \mathrm{mL}) .{ }^{4}$ Interestingly, when incubated with a physiological DNasel concentration $(0.025 \mu \mathrm{g} / \mathrm{mL})$, the mucin-based NPs particles were relatively stable over time: we only detected with a slight size reduction after 3 days, which was accompanied by a moderate decrease in the derived count rate. This suggests that the risk of rapid NP degradation in the bloodstream environment is relatively low.

a

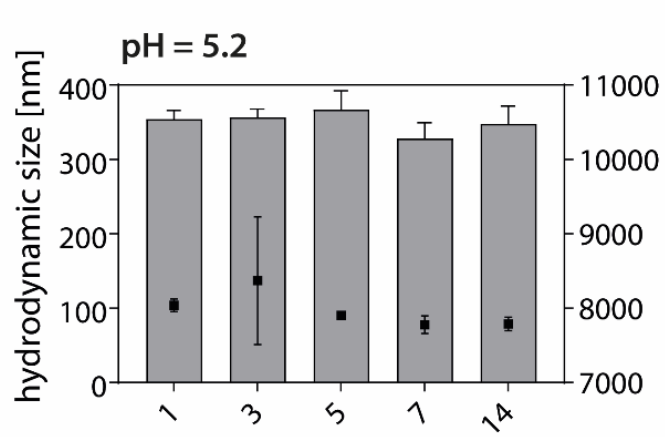

C

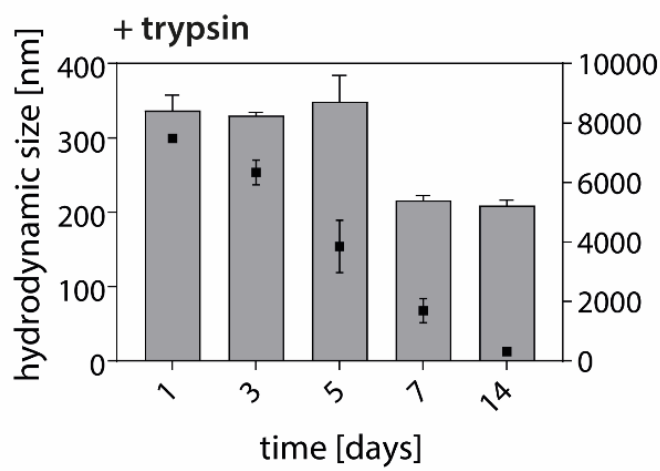

b

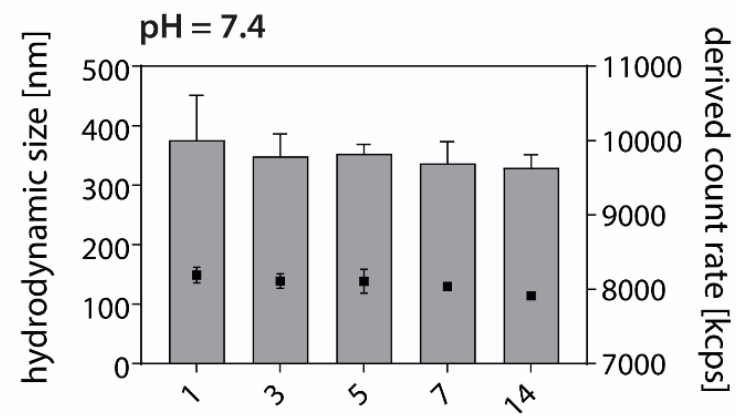

time [days]
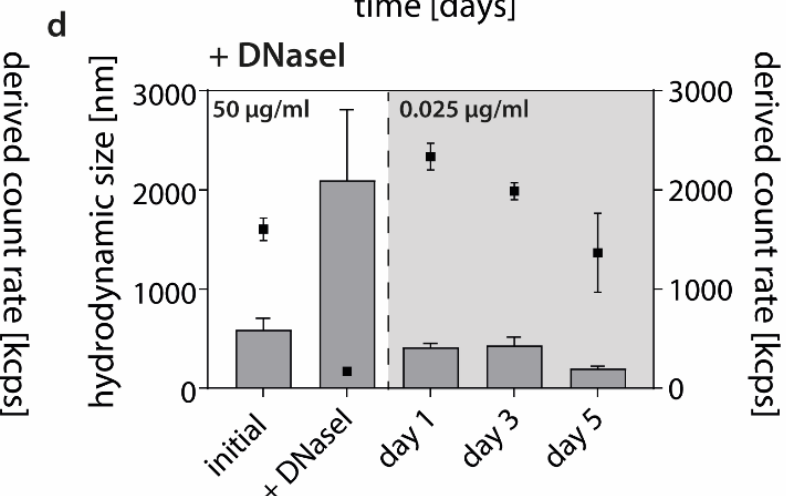

hydrodynamic size [nm]

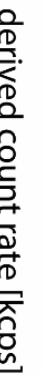

derived count rate $[\mathrm{kcps}]$

Figure S4: Stability of mucin-based NPs at different $\mathrm{pH}$ levels and in the presence of enzymes. $\mathbf{a}, \mathbf{b}$, Hydrodynamic size measurements of mucin-based NPs at $\mathrm{pH}=7.4$ (a) and 5.2 (b). $\mathbf{c}, \mathbf{d}$ Degradation behavior of mucin-based NPs in the presence of trypsin (c), and DNase I at two concentrations (d). Data shown represents mean values, error bars denote the standard deviation as obtained from $n=5$ independent samples.

Table S2: PDI values of mucin NPs that were incubated at different conditions for up to 14 days.

\begin{tabular}{|c|c|c|c|c|c|}
\hline \multirow{2}{*}{ incubation condition } & \multicolumn{5}{|c|}{ PDI value } \\
\cline { 2 - 6 } & Day 1 & Day 3 & Day 5 & Day 7 & Day 14 \\
\hline $\mathrm{pH}=7.4$ & 0.15 & 0.17 & 0.21 & 0.35 & 0.24 \\
\hline
\end{tabular}




\begin{tabular}{|c|c|c|c|c|c|}
\hline $\mathrm{pH}=5.2$ & 0.10 & 0.13 & 0.12 & 0.17 & 0.16 \\
\hline trypsin & 0.18 & 0.19 & 0.32 & 0.41 & 0.48 \\
\hline DNase I $(0.025 \mu \mathrm{g} / \mathrm{mL})$ & 0.19 & 0.26 & 0.61 & - & - \\
\hline DNase I $(50 \mu \mathrm{g} / \mathrm{mL})$ & 0.21 & $0.81^{*}$ & - & - & - \\
\hline
\end{tabular}

* data was taken after $4 \mathrm{~h}$

\section{Drug loading and release profiles}

\subsection{Drug release including a terminal drug release event triggered by heating}

After drug release from the mucin NPs was completed, i.e., when a plateau in the release profiles was reached after $\sim 24 \mathrm{~h}$, the NP suspensions were heated up to $75^{\circ} \mathrm{C}$ (which exceeds the melting temperature of the crDNA/crDNA hybridizations) and the final drug concentration was quantified (Fig. S5).

a

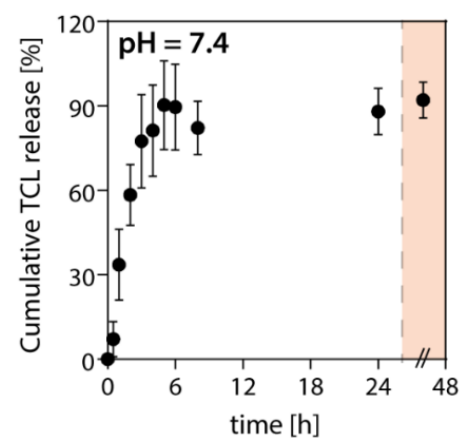

C

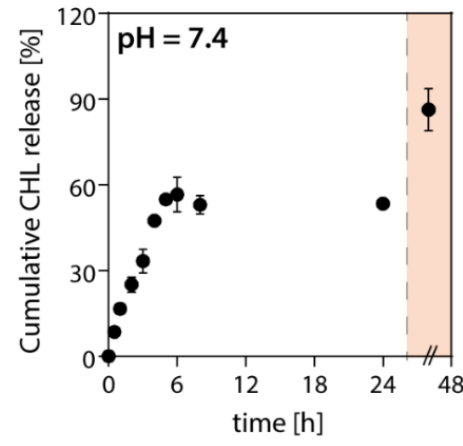

e

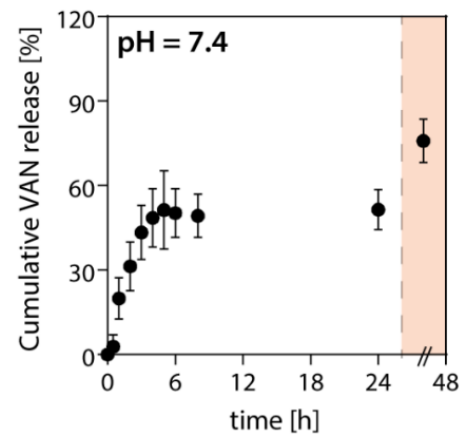

b

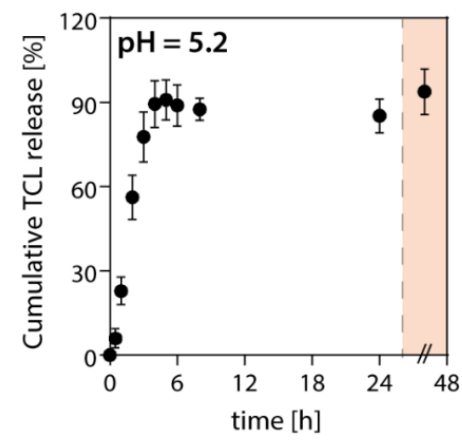

d

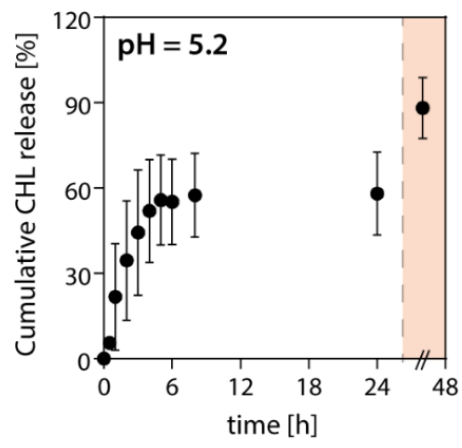

f

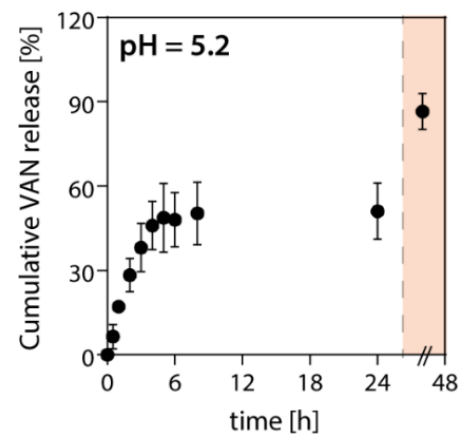


Figure S5: Extended drug release profiles. The data points after the dashed line (red area) show the drug amount detected after subjecting the NPs to a heating step $\left(75^{\circ} \mathrm{C}\right)$. The data shown represents mean values, error bars denote the standard deviation as obtained from $n=3$ independent samples.

\subsection{Encapsulation efficiency of mucin NPs prepared at different salt concentrations}

To check whether the charge state of the drugs is a relevant factor for determining their loading capacity into mucin NPs, we prepared drug loaded NPs in both, physiological buffer (containing $154 \mathrm{mM} \mathrm{NaCl}$ ) and buffer supplemented with $0.5 \mathrm{M} \mathrm{NaCl}$. After dialysis in the corresponding buffers, the drug entrapment efficiency was determined for both cases as described in the main text. Indeed, at the higher salt content, we observed a decrease in the encapsulation efficiency of both, positively and negatively charged drugs (Fig. S6). In contrast, no significant change occurred for the uncharged drug. Therefore, those results suggest that electrostatic interactions between drug molecules and mucin contribute to the loading capacity of the generated NPs.
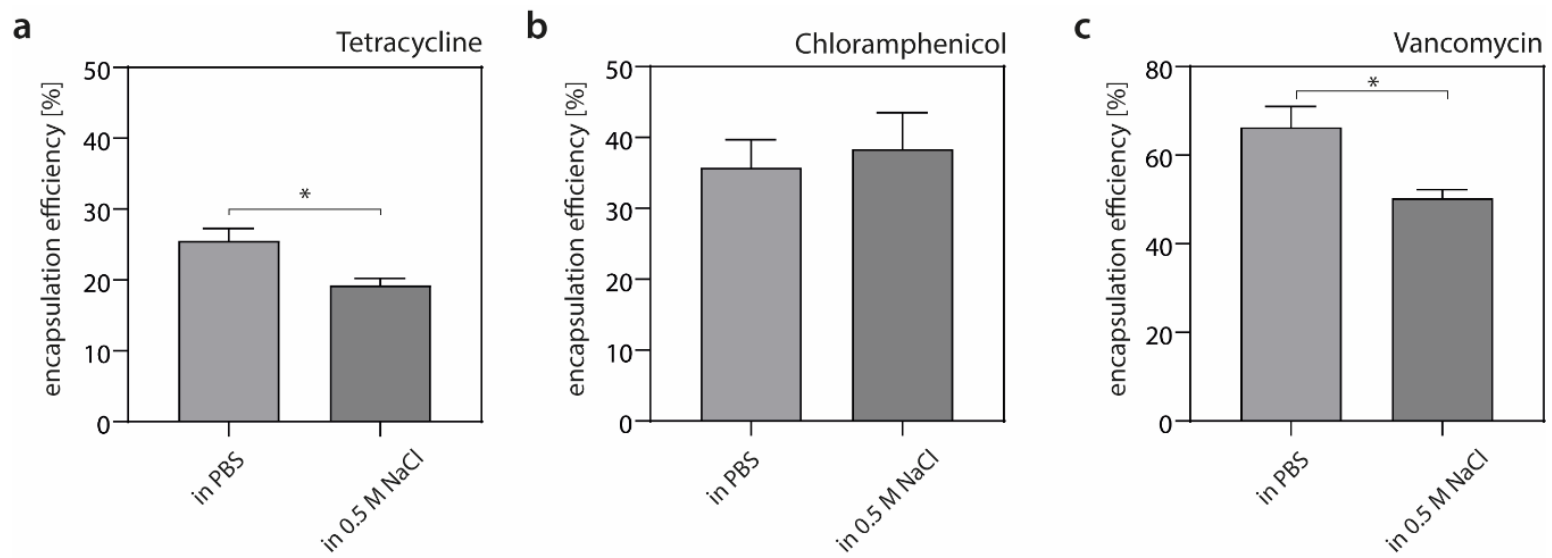

Figure S6: Tetracycline (a), Chloramphenicol (b) and Vancomycin (c) encapsulation efficiency of mucin NPs in different salt conditions. The data shown represents mean values, error bars denote the standard deviation as obtained from $n=5$ independent samples.

\subsection{Determining the minimal dDNA concentration required for triggering drug release from mucin NPs}

Ideally, each dDNA molecule could open a crDNA/crDNA pair. However, we experimentally determined above (Fig. S3) that, at the concentrations used here, 12 dDNA strands per crDNA/crDNA are required. 
To determine the minimum dDNA concentration needed to initiate the conformational change of mucin NPs into elongated objects, we exposed PLL-coated and uncoated mucin NPs to a serially diluted dDNA solution, where the ratio (number of dDNA strands/number of crDNA/crDNA complexes) was adjusted from 0.1 to 10 . Then, the samples were characterized in terms of their hydrodynamic size and PDI (Fig. S7).

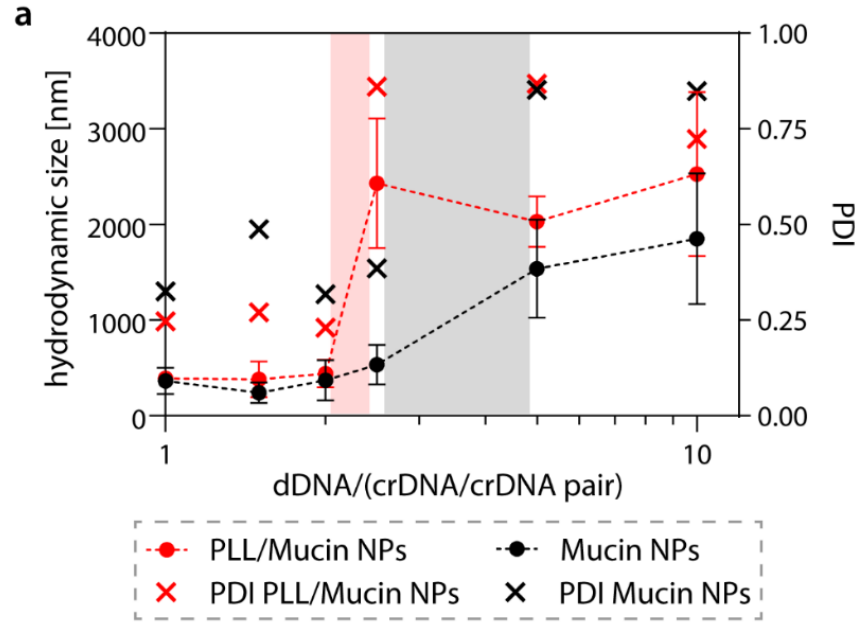

b

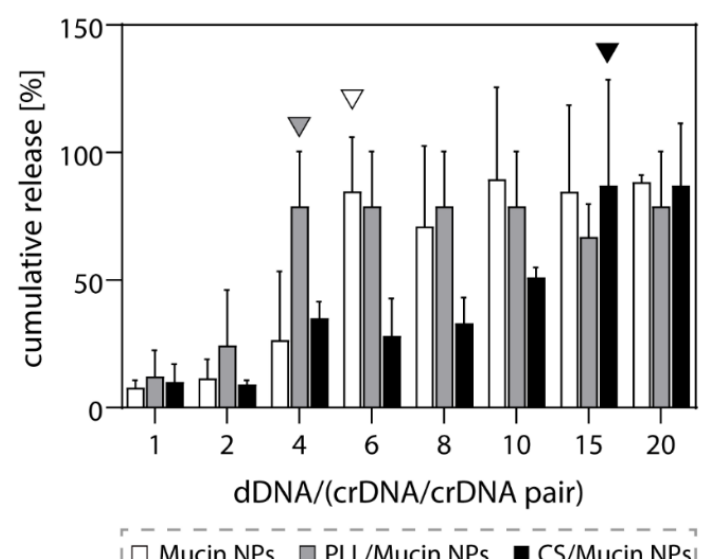

Figure S7: a, Light scattering measurements of mucin NPs under a dDNA gradient showing the threshold dDNA concentration required to force the particulate structure to unfold. $b$, Cumulative drug release from mucin NPs (unmodified, PLL and CS-coated). Triangles show the minimum number of dDNA required to obtain an efficient drug release.

After conducting those DLS experiments, three variants of TCL-loaded NPs (unmodified, CScoated, and PLL-coated) were prepared, and drug release was triggered from these samples by adding different concentrations of dDNA strands (1-20 dDNA molecules per crDNA/crDNA cross-link). The obtained cumulative drug release was quantified after $6 \mathrm{~h}$ of incubation at RT. 
PLL-coated NPs required a lower amount of dDNA strands to obtain a cumulative release of at least 50\% than uncoated mucin-based NPs. In contrast, CS-coated NPs required a larger concentration of dDNA (i.e., 15 dDNA strands per crDNA/crDNA crosslink) to obtain a similarly high level of drug release as for the other two mucin NP variants. This finding can be explained by the strong electrostatic interactions occurring between the polycationic CS coating and the negatively charged DNA strands. 


\section{In vitro cell experiments with mucin NPs}

\subsection{In vitro cytotoxicity of mucin NPs}

The viability of HeLa, NIH/3T3 and THP-1 derived macrophage type 0 after incubation with different mucin NPs (see main text) was evaluated with a WST-1 assay. Cells incubated with only cell culture medium were used as a negative control (NC). First, cells from three separate well plates ( $5 \times 10^{3}$ cells/well of a 96-well plate) were incubated with NPs. After incubating cells with NPs for predetermined time periods (1, 4 and $24 \mathrm{~h}$ for macrophages; $24 \mathrm{~h}$ for HeLa and NIH/3T3 cells). The medium was replaced with a WST-1 solution (10\% v/v in each incubation medium). After an incubation time of $30 \mathrm{~min}$, the absorbance of the medium at $450 \mathrm{~nm}$ was measured with a plate reader (Victor3, Perkin Elmer, Rodgaum, Germany). The cellular viability was calculated by normalizing the absorbance value of NP-treated cells to that obtained for the NC (Fig. S8 for HeLa and NIH/3T3 cells, Fig. 3c for macrophages).

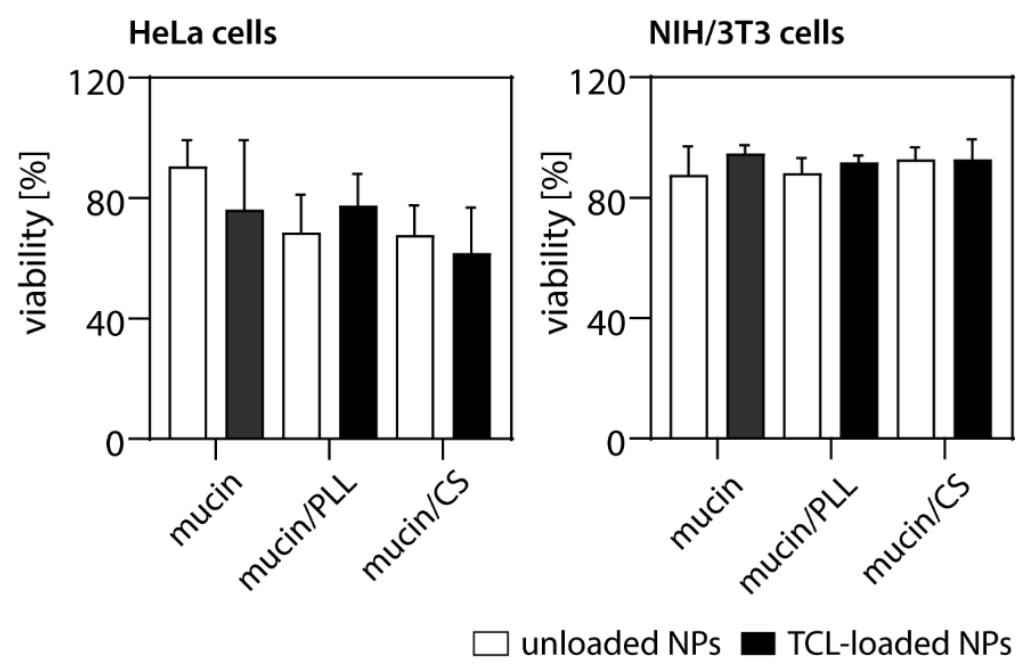

Figure S8: In vitro viability of HeLa and NIH/3T3 cells incubated with mucin NPs for 24 h. Data shown represents mean values, error bars denote the standard deviation as obtained from $n=9$ independent samples.

\subsection{NP internalization verified by FACS}

NPs used for FACS experiments were made from fluorescently-labeled mucins. All FACS measurements were performed in the presence of Trypan Blue (TB) to quench the fluorescence signal arising from NPs bound to the cell membrane. A remarkable increase in 
the fluorescence intensity was observed for the HeLa cells incubated with surface-modified NPs. This demonstrates the successful cellular uptake of those NPs (Fig. S9).

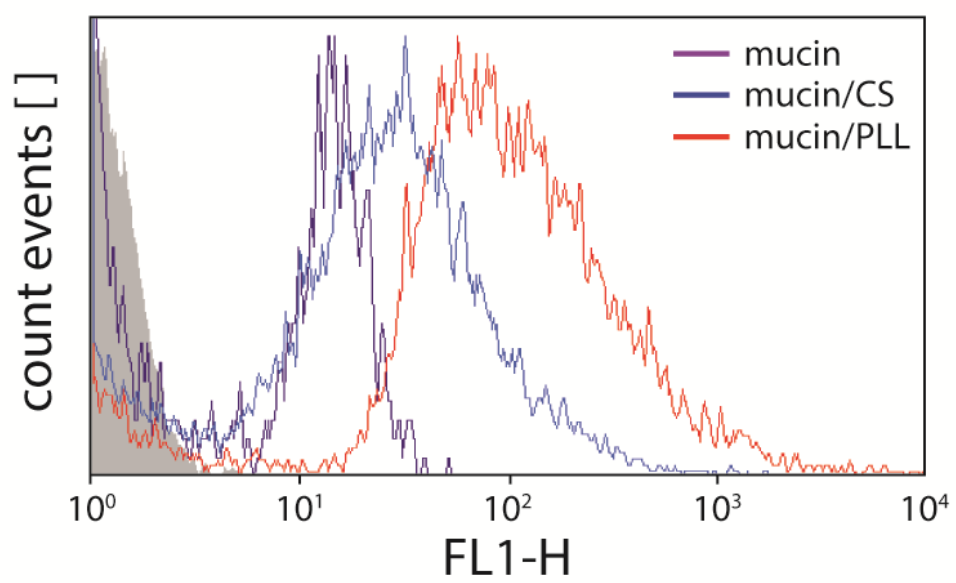

Figure S9: FACS examination. FACS examination of HeLa cells incubated with three different mucin NP variants for 4 h: unmodified mucin NPs, CS-coated mucin NPs and PLL-coated mucin NPs. The grey area represents the control cell population which was not incubated with any mucin NPs.

\subsection{Fluorescence microscopy and CLSM imaging}

NPs used for fluorescence and CLSM imaging were made from fluorescently labeled mucins. HeLa cells were seeded at a density of 6,000 cells/well into a $\mu$-Slide 8-well ibidiTreat plate (ibidi GmbH, Gräfelfing, Germany), covered with $200 \mu \mathrm{L}$ MEM medium (supplemented with $10 \% \mathrm{v} / \mathrm{v}$ FBS, $2 \mathrm{mM}$ L-glutamine and $1 \% \mathrm{v} / \mathrm{v}$ non-essential amino acid solution) and incubated for $24 \mathrm{~h}$. Then, one-fourth of the culture medium (i.e., $50 \mu \mathrm{L}$ ) was replaced with a mucin NP solution, and the NPs were allowed to interact with the cells for $4 \mathrm{~h}$. Then, the cells were washed twice with DPBS and fixed with 4\% formalin. 4,6-diamino-2-phenylindole (DAPI, Sigma Aldrich; 300 nM in PBS) was used for nucleus staining. The images shown in Fig. S10a were acquired on a DMi8 Leica microscope (Leica, Wetzlar, Germany) using a 20x lens (PL Fluotar 20x/0.70, Leica) and a digital camera (Orca Flash 4.0 C11440, Hamamatsu, Japan).

A putative colocalization of NPs and cellular endosomes was tested with CLSM. After $24 \mathrm{~h}$ of cell cultivation, one-fourth of the medium (i.e., $50 \mu \mathrm{L}$ ) was replaced with the mucin NP solution containing $1 \mu \mathrm{L}$ of endosome marker (FM 4-64; Invitrogen T13320, $10 \mu \mathrm{g} / \mathrm{mL}$ in $\left.\mathrm{ddH}_{2} \mathrm{O}\right)$, and the cells were further incubated for additional $24 \mathrm{~h}$. Next, the cells were washed twice with DPBS, fixed with $4 \%$ formalin. Confocal imaging was performed on a Leica TCS 
SP5 II setup (Leica, Wetzlar, Germany) comprising a DMI6000 microscope corpus (Leica) and a 40x oil immersion objective (HCX PL APO; NA = 1.25, Leica). An argon laser (a laser power of $40 \%$ was selected) was used to excite fluorescence from the atto488-labeled mucin NPs at $488 \mathrm{~nm}$, while the endosomes were detected at wavelengths between $590-750 \mathrm{~nm}$. High-resolution images of the cells were analyzed with the software ImageJ (Fiji). The green fluorescent spots in Fig. S10b represent mucin NPs that were able to escape endosomes, whereas signals obtained from NPs trapped in endosomes overlap with the endosomal marker and thus give a yellow/orange color when the images are merged. We speculate that this good result is, at least in part, due to the cationic surface of the PLL-coated mucin NPs. ${ }^{5}$

a

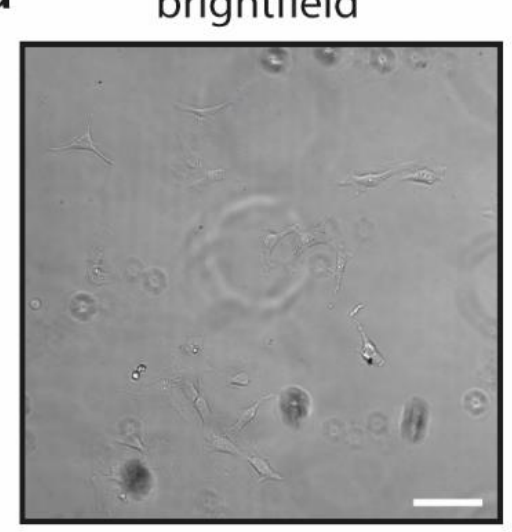

b

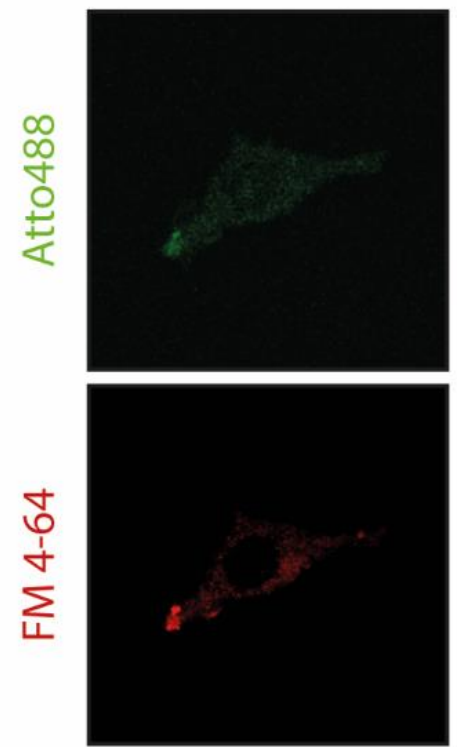

$\mathrm{DAPI}+$ Atto488

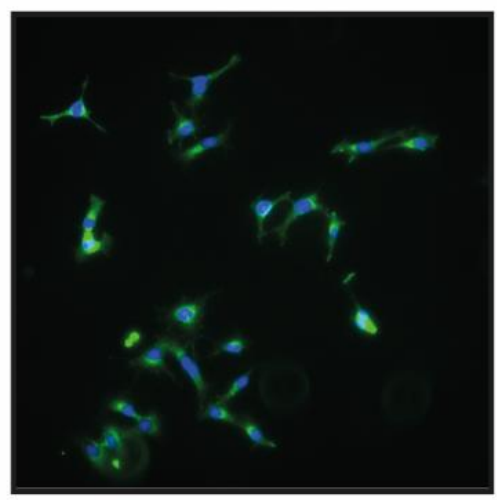

merge

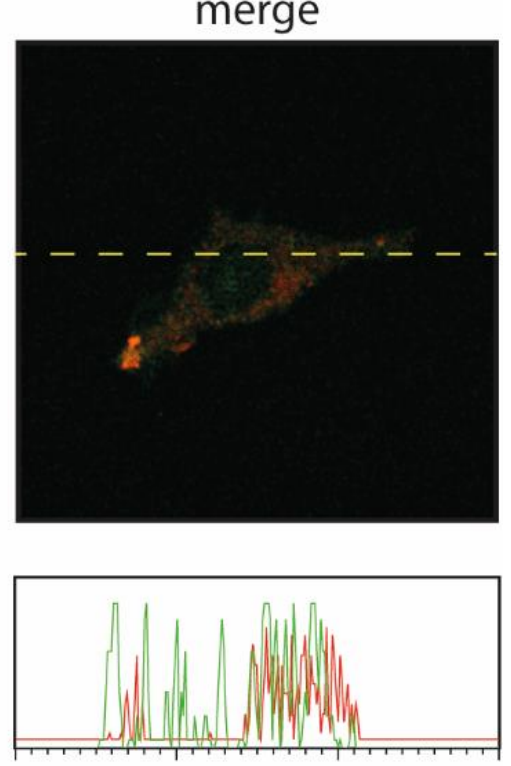

Figure S10: Localization of fluorescently labeled mucin/PLL NPs in HeLa cells. a, Fluorescence microscopy images of HeLa cells incubated with NPs (green) for $24 \mathrm{~h}$. Cell nuclei were stained with DAPI (blue). The scale bar shown in (a) represents $20 \mu \mathrm{m}$ and applies to (b) as well. b, CLSM image of a HeLa cell where endosomes 
are stained with FM4-64 (red) and the NPs are stained with Atto488 (green). The profile in the lower right corner denotes the fluorescence intensity variations measured along the yellow dashed line; the red line represents the location of endosomes and the green line represents mucin NPs.

\section{4. $I C_{50}$ value determination for $T C L$}

The half-maximal inhibitory concentration of TCL $\left(\mathrm{IC}_{50}\right)$ was assessed with a water-soluble tetrazolium (WST-1) assay (Sigma Aldrich). In brief, TCL solutions ( $c=0.1-100 \mathrm{mM}$ ) were incubated with HeLa cells ( $5 \times 10^{3}$ cells/well of a 96-well plate) for $24 \mathrm{~h}$ and the medium in the wells was replaced with a solution containing $20 \mu \mathrm{L}$ WST-1 per $1 \mathrm{~mL}$ medium. After 30 min of incubation, the absorption of the medium was measured at an excitation wavelength of $450 \mathrm{~nm}$ (Victor3 plate reader, Perkin Elmer, Rodgaum, Germany).

Then, the cell viability was calculated by normalizing the absorbance value obtained for drug-treated HeLa cells to that obtained for the negative control (where cells were only incubated with cell culture medium). The cell viability was then plotted against the concentration of the initial drug feed, and the half-maximal growth inhibition $\left(\mathrm{IC}_{50}\right)$ was determined to be $8.4 \mathrm{mM}$, i.e., where the cells showed 50\% viability (Fig. S11).

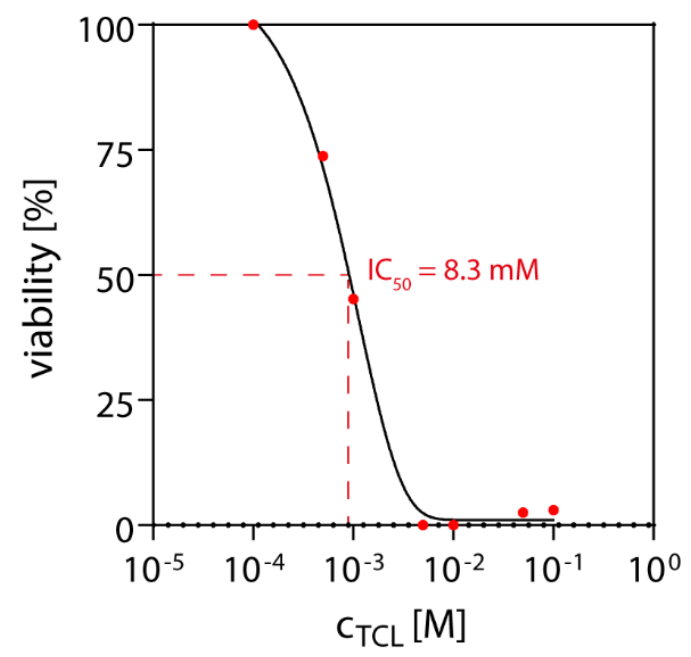

Figure S11: Concentration-dependent cytotoxicity of TCL towards HeLa cells. The TCL concentration corresponding to the viability of HeLa cells of $50 \%$ is then selected as the $\mathrm{IC}_{50}$ value of TCL.

\section{Preparation of DOX-loaded mucin NPs for autonomous drug release in target cells}


Prior to in vitro cell culture experiments, DOX-loaded nanoparticles were characterized in terms of drug encapsulation and loading capacity, on-demand configurational change and release profiles at $\mathrm{pH}=5.2$ and 7.4 (Fig. S12). First, drug loading experiments were carried out as described in the Methods section in a DOX feed range of $0.01-0.09 \mathrm{mg} / \mathrm{mL}$ (Fig. S12a). The on-demand reconfiguration of the DOX-loaded NPs was determined by measuring the change in their hydrodynamic size after addition of a dDNA (Fig. S12b). In these experiments, coDNA was used as a control.

To determine the release profiles at $\mathrm{pH}=5.2$ and $7.4,5 \mathrm{~mL}$ of DOX-NP suspensions were placed in dialysis tubes and the release is initiated by adding $50 \mu \mathrm{L}$ of a $100 \mathrm{mM}$ dDNA solution (Fig. S12c,d). Next, the DOX content of the NPs were determined.

a

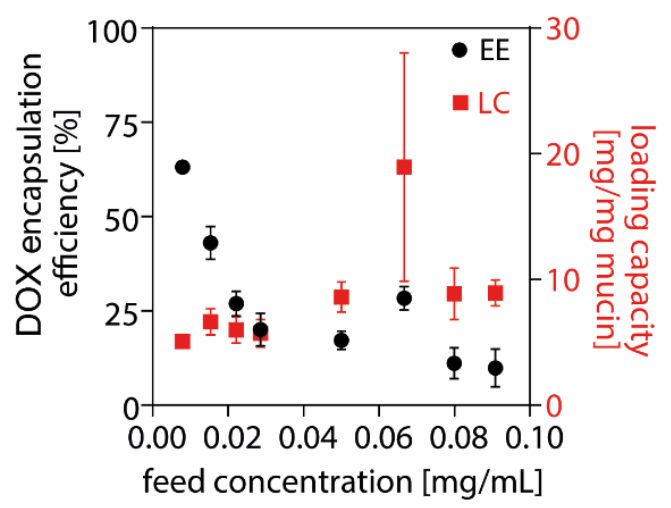

C

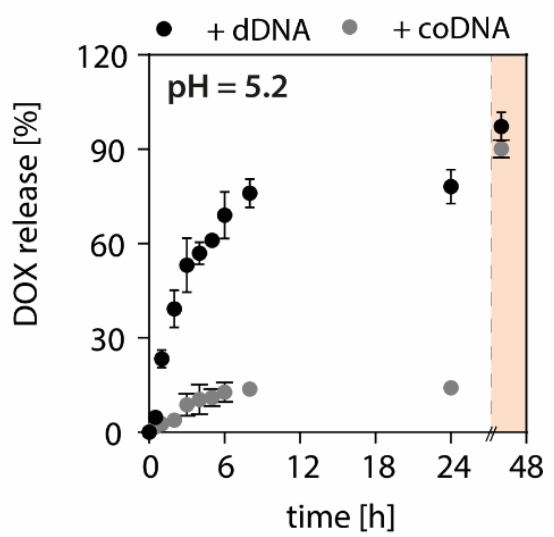

b

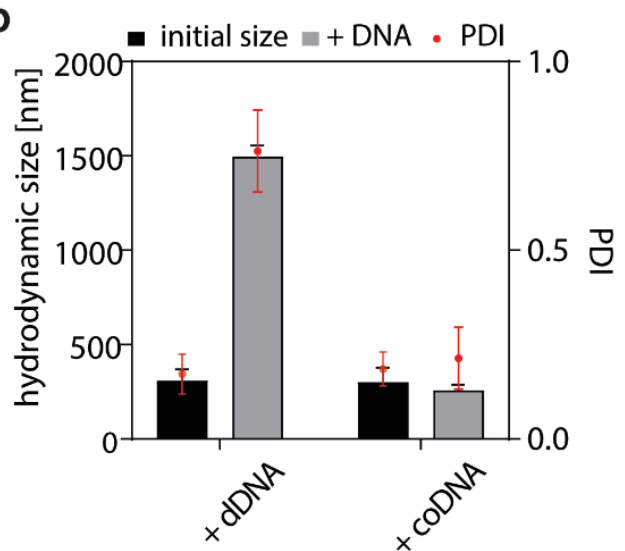

d

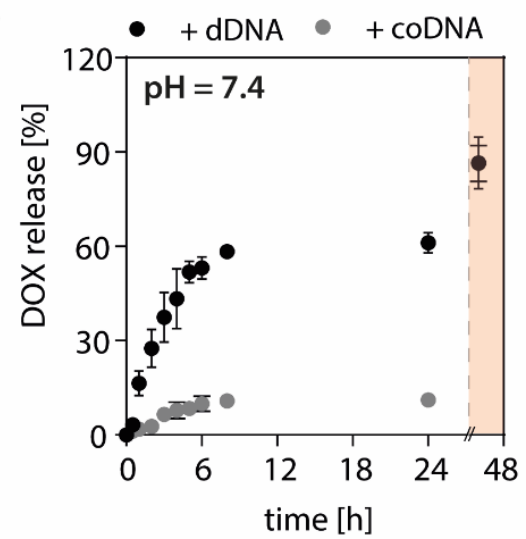

Figure S12: $\mathbf{a}$, DOX encapsulation efficiency and loading capacity of mucin NPs $(n=3) . \mathbf{b}$, On-demand opening of DOX-loaded mucin NPs $(n=5)$ is not possible with the addition of a random control DNA (coDNA), but a trigger DNA (dDNA). $\mathbf{c}, \mathbf{d}$, The cumulative release profiles of DOX-loaded mucin-based NPs $(n=3)$ at $\mathrm{pH}=5.2$ (c) and 7.4 (d). The data points after the dashed line (red area) show the drug amount detected after subjecting the NPs to a heating step $\left(75^{\circ} \mathrm{C}\right)$. The data shown represents mean values, error bars denote the standard deviation. 


\section{Autonomous drug release triggered by intracellular microRNA}

\subsection{Real time-PCR}

Expected differences in miR-21 expression levels were confirmed for the two cell lines used in this study, i.e., HeLa (target cells) and NIH/3T3 (control cells), with RT-PCR. First, HeLa and $\mathrm{NIH} / 3 \mathrm{~T} 3$ cells were cultured to reach approximately $95 \%$ confluency as described in the Methods section. The total RNA content of the cells was extracted (GeneJet RNA Purification Kit, Thermo Fisher), and the RNA concentration was determined using a Nanodrop spectrophotometer. $2000 \mathrm{ng}$ of total RNA was reverse-transcribed into cDNA by using HighCapacity cDNA Reverse Transcription Kit (Thermo Fisher) combined with miR-21 and U6 stem-loop primers. Both stem-loop RT primers were designed according to the method developed by Chen et al. ${ }^{6}$

5'-GTCGTATCCAGTGCAGGGTCCGAGGTATTCGCACTGGATACGACTCAACA-3

(miR-21 stem-loop)

\section{5'-GTCGTATCCAGTGCAGGGTCCGAGGTATTCGCACTGGATACGACAAAATA-3’}

(U6 stem-loop)

Following CDNA synthesis, RT-PCR was performed using primers designed in the studies by Xu et al. and Zhang et al.:7,8

5'-CTCGCTTCGGCAGCACA-3’ (forward U6)

5'-AACGCTTCACGAATTTGCGT-3' (reverse U6)

5'-GCCCGCTAGCTTATCAGACTGATG-3' (forward miR-21)

5'-CAGTGCAGGGTCCGAGGT-3' (reverse miR-21)

RT-PCR was carried out using the PowerUp SYBR Green Master Mix (Applied Biosystems, Thermo Fisher, Waltham, MA, USA) in a StepOnePlus Real-Time PCR System (Thermo Fisher). Amplification signals were observed between the cycles 15 and 35 . U6 was used as an internal control to normalize the miRNA-21 levels. 


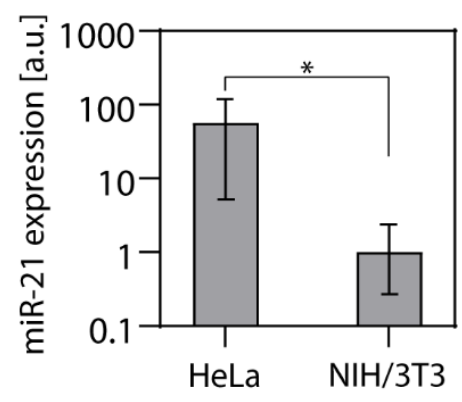

Figure S13: Quantitative real-time PCR analysis of miR-21 in HeLa and NIH/3T3 cells. Data shown represent mean values, error bars denote the standard deviation as obtained from $n=3$ technical replicates.

\subsection{Intracellular drug release experiments}

A series of control experiments were performed to verify that the release of TCL from mucin NPs is highly specific, i.e., that it is only triggered by the correct DNA-base pairing. First, TCLloaded NPs were incubated with HeLa or NIH/3T3 cells for $4 \mathrm{~h}$ as described in the main paper (See "Intracellular drug release" in the Methods section). Next, cells were transfected with either miR-21 mimics (i.e., trigger strand) or coDNA. To quantify the amount of liberated TCL, cytosol fractions of cell lysates were collected as described in the main text, and their TCL content was determined spectrophotometrically (Fig. S14, black bars). Then, those cytosol fractions were subjected to a dialysis step (see main text), and the remaining TCL amount was determined (Fig. S14, grey bars). With this procedure, a significant reduction in the TCL amount was achieved for HeLa as well as NIH/3T3 cells transfected with miR-21 mimics; this shows that, here, free (= liberated) TCL was present in the cytosol. In contrast, no significant reduction was observed for the control group of NIH/3T3 cells, where coDNA was transfected (Fig. S14, d).

Importantly, for HeLa cells, which produce miR-21 strands, our results indicated the successful release of TCL from the mucin NPs even without transfection of the correct trigger DNA strands (Fig. S14, b) - albeit with lower efficiency than when the miR-21 mimics are supplied externally. This agrees with our assumption that cytosolic miR-21 strands produced by the HeLa cell line should be sufficient to open the mucin NPs. At the same time, it shows that this autonomous liberation of TCL from mucin NPs is - at least to some extent - cellspecific. 
a

HeLa cells

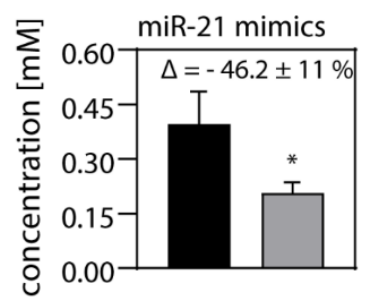

$\mathrm{NIH} / 3 \mathrm{~T} 3$ cells

C

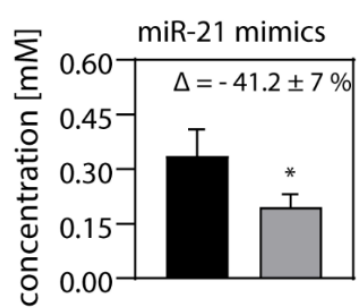

initial TCL concentration b

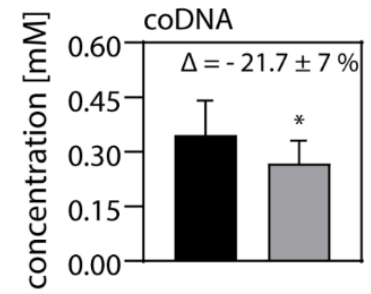

d

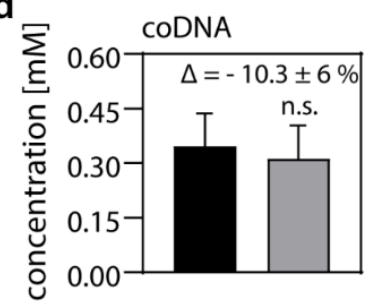

TCL concentration after dialysis

Figure S14: TCL concentration in the cytosol fractions of HeLa and NIH/3T3 cells, respectively, before (black bars) and after dialysis (grey bars). Cells are transfected with either miR-21 mimics or coDNA, as defined on top of each graph. Insets show the percentage of TCL concentration reduction in the cytosols achieved by cytosol dialysis and indicates the liberated drug amount. Data shown represent mean values, error bars denote the standard deviation as obtained from $n=6$ independent samples.

\section{Fourier-transform infrared spectroscopy (FT-IR)}

Mucin/DNA mixtures were prepared as described in the main text and pre-freezed at $-20^{\circ} \mathrm{C}$. Next, the samples were lyophilized for 1 day and analyzed with Fourier Transform Infrared Spectroscopy (FT-IR, Vertex 70 FTIR, Bruker, Germany) in the wavelength range of 4000 to $700 \mathrm{~cm}^{-1}$.

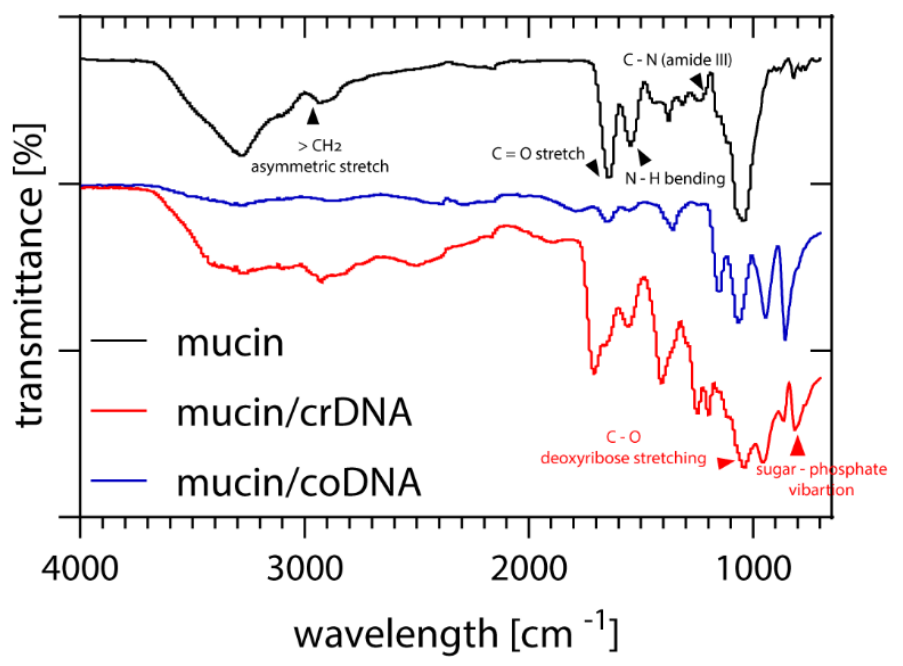

Figure S15: FT-IR diagram of mucin, mucin/crDNA and mucin/coDNA 


\section{Calibration curves}

Standard curves for the three drug solutions shown (Figure S16) were obtained by measuring the absorbances of serially diluted drug solutions (at $280 \mathrm{~nm}$ for CHL and VAN, at $360 \mathrm{~nm}$ for TCL and at $450 \mathrm{~nm}$ for DOX, respectively) with a spectrophotometer (specord 210, Analytikjena, Jena, Germany). In the concentration range tested here, a linear relation between the absorbance values and the drug concentration holds.

a

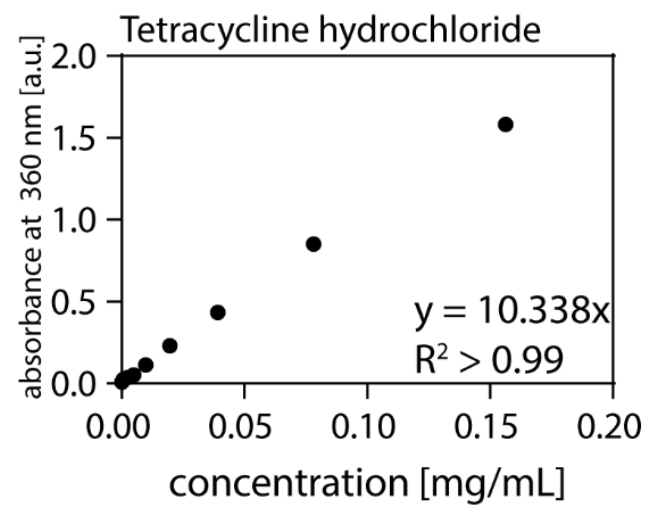

C

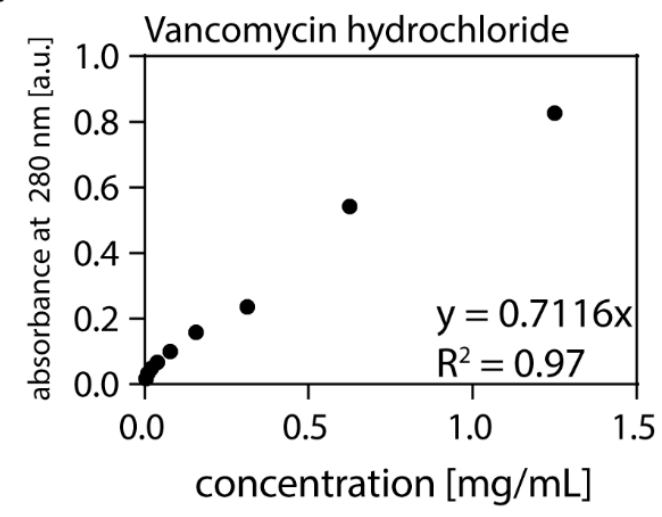

b

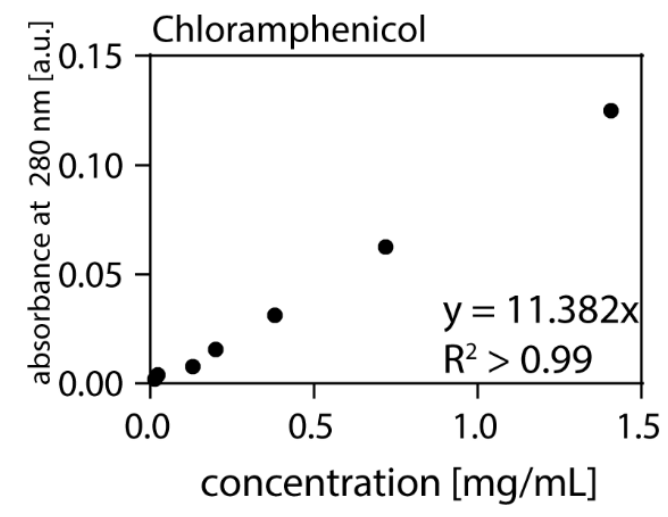

d

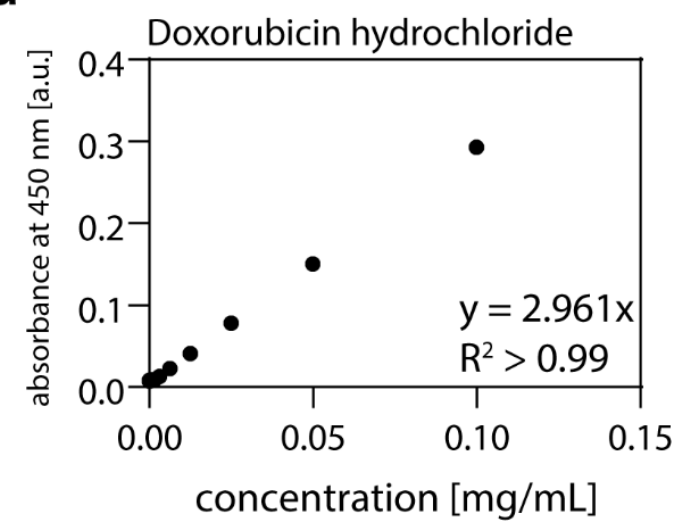

Figure S16: Standard curves. a, Tetracycline hydrochloride $(\mathrm{TCL}) \mathbf{b}$, chloramphenicol (CHL) c, vancomycin hydrochloride (VAN) and d, Doxorubicin standard curves which relate the absorbance of the respective drug solution and with the drug concentration.

\section{DNA sequences}

For the synthetic DNA sequences used in this study, the energy needed to fully break the structures formed with either crDNA, or antimir-21 $(\Delta \mathrm{G})$ was calculated by considering the longest stretch of complementary bases. Also, oligonucleotides used as crosslinkers were tested with the NUPACK software to ensure that they do not form any secondary structures 9 . The minimum free energy of the structures was calculated using nearest-neighbor empirical 
parameters for oligonucleotides at $37^{\circ} \mathrm{C}$ and in the presence of $5 \mathrm{mM} \mathrm{Mg}^{2+}$ and $150 \mathrm{mM} \mathrm{Na}$.

Table S3 and Table S4 list the molecular design of the sequences and their properties such as dimerization energy, melting temperature, the number of hybridized base pairs and modifications.

Table S3: Designed oligonucleotide sequences. The table lists their dimerization energy, melting temperature and the possible number of hybridized base pairs when they interact with crDNA strands.

\begin{tabular}{|c|c|c|c|c|c|}
\hline abbreviation & sequence from $5^{\prime}$ to $3^{\prime}$ & $\begin{array}{c}\text { dimerization } \\
\text { energy, } \Delta \mathrm{G} \\
(\mathrm{kcal} / \mathrm{mol})\end{array}$ & $\begin{array}{c}\text { melting } \\
\text { tempera- } \\
\text { ture }\left({ }^{\circ} \mathrm{C}\right)\end{array}$ & $\begin{array}{c}\text { \# of } \\
\text { hybridized } \\
\text { base pairs }\end{array}$ & $\begin{array}{c}\text { modification } \\
\text { at the } 5^{\prime} \text { end }\end{array}$ \\
\hline crDNA & AAAAGAAGCAAAGACAACCCGGGTAA & -18.57 & 60.6 & 8 & thiol \\
\hline dDNA & TTACCCGGGTTGCTTGCTC & -43.99 & 75.3 & 22 & - \\
\hline CODNA & AATGAGCACAACAGAAACGAAG & -3.61 & N/A & 2 & - \\
\hline
\end{tabular}

Table S4: Designed oligonucleotide sequences for the HeLa-cell specific release experiments. The table lists their dimerization energy, melting temperature and the possible number of hybridized base pairs when they interact with antimir-21 strands.

\begin{tabular}{|c|c|c|c|c|c|}
\hline abbreviation & sequence from 5'to 3' & $\begin{array}{c}\text { dimerization } \\
\text { energy, } \Delta \mathrm{G} \\
(\mathrm{kcal} / \mathrm{mol})\end{array}$ & $\begin{array}{c}\text { melting } \\
\text { tempera- } \\
\text { ture }\left({ }^{\circ} \mathrm{C}\right)\end{array}$ & $\begin{array}{c}\# \text { of } \\
\text { hybridized } \\
\text { base pairs }\end{array}$ & $\begin{array}{c}\text { modification } \\
\text { at the } 5^{\prime} \text { end }\end{array}$ \\
\hline antimiR-21 & TCAGTCTGATATCA & -10.97 & 48.2 & 8 & thiol \\
\hline $\begin{array}{c}\text { miR-21 } \\
\text { mimics }\end{array}$ & TATCAGACTGA & -15.61 & 39.0 & 11 & - \\
\hline coDNA-2 & GGAATCCAAGTG & -1.95 & $\mathrm{~N} / \mathrm{A}$ & 2 & - \\
\hline
\end{tabular}

\section{References}

1. Schömig, V. J.; Käsdorf, B. T.; Scholz, C.; Bidmon, K.; Lieleg, O.; Berensmeier, S. An Optimized Purification Process for Porcine Gastric Mucin with Preservation of Its Native Functional Properties. RSC Adv. 2016, 6, 44932-44943.

2. Gaumet, M.; Vargas, A.; Gurny, R.; Delie, F. Nanoparticles for Drug Delivery: The Need for Precision in Reporting Particle Size Parameters. Eur. J. Pharm. Biopharm. 2008, 69, 1-9.

3. Käsdorf, B. T.; Weber, F.; Petrou, G.; Srivastava, V.; Crouzier, T.; Lieleg, O. Mucin-Inspired Lubrication on Hydrophobic Surfaces. Biomacromolecules 2017, 18, 2454-2462.

4. Macanovic, M.; Lachmann, P. Measurement of Deoxyribonuclease I (DNAse) in the Serum and Urine of Systemic Lupus Erythematosus (SLE) - Prone NZB/NZW Mice by a New Radial Enzyme Diffusion Assay. Clin. Exp. Immunol. 1997, 108, 220-226.

5. Kircheis, R.; Kichler, A.; Wallner, G.; Kursa, M.; Ogris, M.; Felzmann, T.; Buchberger, M.; Wagner, E. Coupling of Cell-Binding Ligands to Polyethylenimine for Targeted Gene Delivery. Gene Ther. 1997, 4, 409-418.

6. Chen, C.; Ridzon, D. A.; Broomer, A. J.; Zhou, Z.; Lee, D. H.; Nguyen, J. T.; Barbisin, M.; Xu, N. L.; Mahuvakar, V. R.; Andersen, M. R. Real-Time Quantification of microRNAs by Stem - Loop RT PCR. Nucleic Acids Res. 2005, 33, e179.

7. Xu, X. M.; Qian, J. C.; Deng, Z. L.; Cai, Z.; Tang, T.; Wang, P.; Zhang, K. H.; Cai, J.-P. Expression of Mir-21, Mir-31, Mir-96 and Mir-135b Is Correlated with the Clinical Parameters of Colorectal Cancer. Oncol. Lett. 2012, 4, 339-345. 
8. Zhang, P.; He, Z.; Wang, C.; Chen, J.; Zhao, J.; Zhu, X.; Li, C.-Z.; Min, Q.; Zhu, J.-J. In Situ Amplification of Intracellular microRNA with MNAzyme Nanodevices for Multiplexed Imaging, Logic Operation, and Controlled Drug Release. ACS Nano 2015, 9, 789-798.

9. Zadeh, J. N.; Steenberg, C. D.; Bois, J. S.; Wolfe, B. R.; Pierce, M. B.; Khan, A. R.; Dirks, R. M.; Pierce, N. A. Nupack: Analysis and Design of Nucleic Acid Systems. J. Comput. Chem. 2011, 32, 170-173. 